\title{
Analysis and Improvement on A Lightweight Two-factor Authentication Scheme for Wireless Body Area Networks in Health-care IoT
}

\author{
Kaiqiang Mai, Baoyuan Kang, Rui Li \\ School of Computer Science and Technology, Tiangong University, Tianjin 300387, China
}

\begin{abstract}
Recently, medical and healthcare associations have gradually begun to use the Internet of Things and wireless sensor networks to monitor, collect data, and use wireless body area networks (WBANs) to communicate with patients. However, due to the characteristics of wireless networks that can freely access data on public channels, WBANs face some problems in the security and privacy protection. At the same time, some authentication schemes for wireless body area networks have also been proposed. In this paper, we carefully analyze Fotouhi et al.'s authentication scheme for wireless body area networks in health-care IoT and find that Fotouhi et al.'s scheme is vulnerable to several weaknesses. The main shortcoming of Fotouhi et al.'s scheme is that it takes up too much storage space of each entity during the registration and authentication phase. To overcome the shortcomings of Fotouhi et al.'s scheme, we propose an improved scheme. In our improved scheme, each entity will store less data than Fotouhi et al.'s scheme in the registration phase. Furthermore, we make the gateway node store as little data as possible by adding some key information to the transmitted messages in the authentication phase of the improved scheme. These measures can greatly save a lot of storage space and improve efficiency. We also investigate the security of the improved scheme in informal analysis and formal analysis.
\end{abstract}

Keywords: WBAN, Healthcare, Authentication scheme, Safety.

\section{Introduction}

The wireless body area network (WBAN) can perform real-time medical care monitoring and support through various intelligent medical sensors in or around the patient. These networks are especially helpful for elderly people who need long-term care and do not need to be hospitalized. However, the security of messages transmitted between patients and doctors is also an issue. An adversary can eavesdrop, intercept, modify, or replay data stored in mobile devices or sensors. Therefore, it is very important to ensure the security of transmitted messages. In addition, due to the power limitation of sensor nodes in the human body, efficiency and lightweight are also challenges for WBANs.

Recently, some authentication schemes to the security and privacy issues in WBANs have been proposed [1-14]. Fotouhi et al. [15] proposed a lightweight two-factor authentication scheme and claimed that the proposed protocol can meet the perfect forward security, key compromise impersonation resiliency, and other security requirements for health-care authentication schemes. We find the application scenarios of Fotouhi et al.'s scheme is different from common authentication schemes for WBANs [1-14]. In Fotouhi et al.'s scheme there are three kind participants, a gateway, sensors and users. The gateway, a sensor and a user want share a session key. But, the sensor and the user cannot communicate directly. It is undeniable that Fotouhi et al.'s scheme can resist the replay attacks and tracking attacks by updating the temporary identities of users and sensors, which also avoid the verification of timestamps.

However, in this paper, we find that Fotouhi et al.'s scheme is vulnerable to several weaknesses. Firstly, there is too much data storage in registration phase. Secondly, some identities data missing in some submitted messages in authentication phase. Finally, more data are needed stored in authentication phase. So, Fotouhi et al.'s scheme is difficult to execute effectively and will take up a lot of storage space. On the basis of analyzing to Fotouhi et al.'s scheme, we propose an improvement on Fotouhi et al.'s scheme. In the registration phase of the improved scheme, each of our entities will store less data and ensure that there are no missing messages in the messages transmitted between entities. Meanwhile, in the authentication phase the gateway node store as little data as possible. We also investigate the security of the improved scheme in informal analysis and formal analysis.

The rest of the paper is organized as follows. In Section 2, we briefly review Fotouhi et al.'s scheme and analyze its weaknesses in Section 3. The improved protocol is proposed in Section 4. Security cryptanalysis is given in Section 5. Finally, the article is concluded in Section 6.

\section{Review of Fotouhi et al.'s Scheme}

In this section, we review Fotouhi et al.'s [15] authentication scheme for wireless body area networks in health-care IoT. Fotouhi et al.'s scheme consists of four phases: Initialization, registration, login and authentication, and the password change phase. Here, we only review the first three phases. Table 1 lists all the notations used in this paper.

Table 1: Notations and their importance

\begin{tabular}{|c|c|}
\hline Symbol & Description \\
\hline$U_{i}, I D_{i}, P W_{i}$ & user and her/his identity and password respectively \\
\hline$G I D_{\mathrm{j}}, G_{\mathrm{j}}$ & $\begin{array}{c}\text { identity and secret key of jth gateway } \mathrm{GWj}, \\
\text { respectively }\end{array}$ \\
\hline$S N_{k}, S I D_{k}$ & kth sensor and its identity respectively \\
\hline$N_{1}$ & number of the sensor set \\
\hline$S G_{k}$ & $\begin{array}{c}\text { shared secret key between sensor and the } \\
\text { corresponding gateway }\end{array}$ \\
\hline$s k_{u}, s k_{g}, s k_{s}$ & $\begin{array}{c}\text { common session keys generated by the user, gateway } \\
\text { and sensor }\end{array}$ \\
\hline$M_{i}$ & messages in authentication phase \\
\hline
\end{tabular}




\begin{tabular}{|c|c|}
\hline$C I D_{i}, Q I D_{k}$ & $\begin{array}{c}\text { user's and sensor's temporary pseudonyms } \\
\text { respectively }\end{array}$ \\
\hline$R_{u}, R_{g}, R_{s}, R_{x}, R_{y}, R_{z}$ & temporary random numbers \\
\hline$h()$. & collision-resistant cryptographic one-hash function \\
\hline$\oplus$ & exclusive-or operation \\
\hline$\|$ & concatenation operation \\
\hline
\end{tabular}

\subsection{Initialization Phase}

In this phase, assume that each gateway is a trusted party, and every message will be transmitted through the gateways identified with $G I D_{\mathrm{j}}$. Each gateway generates a secret key called $G_{\mathrm{j}}$ and selects a collision-resistant cryptographic hash function $\mathrm{h}($.) to initialize the wireless body area network or networks.

\subsection{Registration Phase for Sensor}

In this phase, each sensor node called $S N_{k}$ has an identifier $S I D_{k}$. And, each set of sensors who belong to same network have same network identifier $\mathrm{N}_{1}$. Also each sensor and the corresponding gateway $G W_{\mathrm{j}}$ communicate with each other directly and share a common secret key $S G_{k}$ which is equal to $h\left(S I D_{k}\left\|G_{j}\right\| N_{1}\right)$, and the following operations are performed:

Step 1. $G W_{\mathrm{j}}$ selects two random numbers $R_{y}$ and $R_{z}$ and a pseudo-identity $Q I D_{k}$ for each sensor.

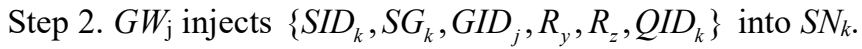

Step 3. $G W_{\mathrm{j}}$ also stores $\left\{S I D_{\mathrm{k}}, Q I D_{k}, N_{1}, R_{y}, h\left(R_{z}\right)\right\}$ in its database. Then, the sensor will be installed in the intended place.

\subsection{Registration Phase for User}

In this phase, the user, $U_{i}$ registers with the gateway, $G W_{\mathrm{j}}$ and the following operations are performed:

Step 1. $U_{i}$ selects an identity, $I D_{i}$ and the corresponding password, $P W_{i}$ and a secret random nonce $R_{0}$ to compute masked password $H P W_{\mathrm{i}}=h\left(P W_{\mathrm{i}} \| R_{0}\right)$ and sends $I D_{i}$ and $H P W_{i}$ to $G W_{\mathrm{j}}$ via a secure channel.

Step 2. If $I D_{i}$ has already been unregistered, $G W_{\mathrm{j}}$ generates a pseudo-identity $C I D_{i}$ and a random number $R_{x}$ for $U_{i}$ and stores them with $I D_{i}$ and $H P W_{i}$ in its database. Then, it computes $A_{1}=h\left(C I D_{i}\left\|R_{x}\right\| G I D_{\mathrm{j}} \| G_{\mathrm{j}}\right) \oplus H P W_{i}$ and $A_{2}=h\left(I D_{i}\right.$ $\left.\| G_{j}\right) \oplus h\left(I D_{i} \| H P W_{i}\right)$ and sends $A_{1}, A_{2}, G I D_{i}, G I D_{\mathrm{j}}$ to $U_{i}$ via a secure channel.

Step 3. $U_{i}$ calculates $A_{3}=h\left(I D_{i} \| P W_{i}\right) \oplus R_{0}$ and stores $A_{1}, A_{2}$, $A_{3}, C I D_{i}, G I D_{i}$ into his/her mobile device. Now $U_{i}$ is ready to be authenticated through an insecure channel as described below.

\subsection{Login and Authentication Phase}

In this phase, $U_{i}, G W_{\mathrm{j}}$, and $S N_{k}$ carry out mutual authentication to set up a session key. The detailed description of the authentication phase are as follows:

Step 1. the $U_{i}$ selects intended sensor and insert $I D_{i}$ and $P W_{i}$ to the mobile device which calculates $R_{0}=A_{3} \oplus h\left(I D_{i} \| P W_{i}\right)$ and $H P W_{i}=h\left(P W_{i} \| R_{0}\right)$. Then, it generates a random number $R_{u}$ and selects sensor's corresponding $S I D_{k}$ and computes $B_{1}=A_{1} \oplus H P W_{i}, B_{2}=B_{1} \oplus H P W_{i} \oplus R_{u}, B_{3}=S I D_{k} \oplus$ $h\left(I D_{i} \| R_{u}\right), B_{4}=h\left(C I D_{i}\left\|G I D_{j}\right\| S I D_{k}\left\|B_{1}\right\| I D_{i} \| R_{u}\right)$. Then, the message $M_{1}$ containing $C I D_{i}, G I D_{\mathrm{j}}, B_{2}, B_{3}$ and $B_{4}$ is sent to $G W_{\mathrm{j}}$.

Step 2. $G W_{\mathrm{j}}$ checks $G I D_{\mathrm{j}}$ and $C I D_{i}$ and fetches the corresponding $I D_{\mathrm{i}}, R_{x}$ and $H P W_{i}$ from its database, if they exist. Then, it computes $B_{1}=h\left(C I D_{i}\left\|R_{x}\right\| G I D_{j} \| G_{j}\right)$ and $R_{u}=B_{2} \oplus B_{1} \oplus H P W_{i}$ and generates two random numbers $R_{g}$ and $R_{z}^{\text {new }}$. Afterwards, $G W_{\mathrm{j}}$ obtains $S I D_{k}$ by calculating $B_{3} \oplus h\left(I D_{i} \| R_{u}\right)$ and gets $R_{y}$ from its database. Then, it generates a new pseudonym $Q I D_{k}^{\text {new }}$ for the sensor. In the following, $G W_{\mathrm{j}}$ computes $S G_{k}=h\left(S I D_{k}\left\|G_{j}\right\| N_{1}\right), S=h$ $\left(S G_{k} \| G I D_{j}\right), B_{5}=\left(R_{u} \oplus H P W_{i}\right) \oplus S \oplus R_{y}, B_{6}=\operatorname{Rg} \oplus S \oplus S I D_{i}$ $\oplus R_{y}, \quad B_{7}=Q I D_{k}^{\text {new }} \oplus R_{g} \oplus R_{y}, \quad B_{8}=h\left(R_{g}\left\|R_{y}\right\| S\right) \oplus R_{z}^{\text {new }}$, $B_{9}=h\left(Q I D_{k}\left\|B_{7}\right\| B_{8}\left\|S G_{k}\right\| \quad R_{u} \oplus H P W_{k} \| R_{g}\right)$ and sends the message $M_{2}=\left(Q I D_{k}, B_{5}, B_{6}, B_{7}, B_{8}, B_{9}\right)$ to the sensor.

Step 3. After receiving the message, the sensor first checks the $Q I D_{k}$ and calculates $S=h\left(S G_{k} \| G I D_{j}\right),\left(R_{u} \oplus H P W_{i}\right)=$ $B_{5} \oplus S \oplus R_{y}$ and $R_{g}=B_{6} \oplus S \oplus S I D_{i} \oplus R_{y}$. Then if $B_{9}$ is correct, it generates a random number $R_{\mathrm{s}}$ and computes $R_{z}^{\text {new }}=h\left(R_{g}\left\|R_{y}\right\| S\right) \oplus B_{8}, \quad Q I D_{k}^{\text {new }}=B_{7} \oplus R_{g} \oplus R_{y}$. After generating $B_{10}=R_{g} \oplus S \oplus R_{z}$, it stores $Q I D_{k}^{\text {new }}, R_{z}^{\text {new }}$ and $R_{y}^{\text {new }}=h\left(R_{y}\right)$ and generates the common session key $S K_{s}=h\left(R_{u} \oplus H P W_{i}\left\|R_{g}\right\| R_{s}\right)$. Finally the sensor generates $B_{11}=h\left(S G_{k} \| R_{g}\right) \oplus h\left(R_{y}\right) \oplus R_{s} \quad, \quad B_{12}=h\left(B_{10}\left\|B_{11}\right\| s k_{s} \|\right.$ $\left.S I D_{k}\left\|G I D_{j}\right\| R_{s}\right)$ and sends $M_{3}=\left(B_{10}, B_{11}, B_{12}\right)$ to the $G W_{\mathrm{j}}$ as the response.

Step 4. On receiving the response from sensor, the $G W_{\mathrm{j}}$ computes $R_{y}^{\prime}=h\left(R_{y}\right)$ in which $R_{y}$ is fetched from DB and $R_{z}^{\prime}=R_{g} \oplus S \oplus B_{10}$.

Then, it checks if $h\left(R_{z}\right) \stackrel{?}{=} h\left(R_{z}^{\prime}\right)$ holds, it calculates $R_{s}=B_{11}$ $\oplus h\left(S G_{k} \| R_{g}\right) \oplus R_{y}^{\prime} \quad$ and obtains the $S K_{g}=h\left(R_{u} \oplus\right.$ $H P W_{i}\left\|R_{g}\right\| R_{s}$ ) as the common session key. Then, it checks $B_{12}$ and generates a new $C I D_{i}$ for $U_{i}$ and stores $Q I D_{k}^{\text {new }}$ and 
$R_{z}^{\text {new }}$. In addition, it replaces the $R_{y}^{\prime}$ with the previous $R_{y}$ and $h\left(R_{x}\right)$ with the $R_{x}$.

In the following, $G W_{\mathrm{j}}$ computes:

$$
\begin{gathered}
B_{13}=h\left(C I D_{i}^{\text {new }}\left\|h\left(R_{x}\right)\right\| G I D_{j} \| G_{j}\right) \oplus h\left(R_{u} \| H P W_{i}\right), \\
B_{14}=h\left(R_{u} \| I D_{i}\right) \oplus R_{g}, \\
B_{15}=h\left(R_{u}\left\|R_{g}\right\| H P W_{i}\right) \oplus R_{s}, \\
B_{16}=h\left(h\left(I D_{i} \| G_{j}\right) \| R_{s}\right) \oplus C I D_{i}^{\text {new }},
\end{gathered}
$$

$B_{17}=h\left(s k_{g}\left\|I D_{i}\right\| B_{13} \| C I D_{i}^{\text {new }}\right)$ and finally forwards them to the $U_{i}$ with the message $M_{4}=\left(B_{13}, B_{14}, B_{15}, B_{16}, B_{17}\right)$.

Step 5. $U_{i}^{\prime} s$ mobile device retrieves $R_{g}$ and $R_{s}$ by calculating, $R_{g}=B_{14} \oplus h\left(R_{u} \| I D_{i}\right), R_{s}=B_{15} \oplus h\left(R_{u}\left\|R_{g}\right\| H P W_{i}\right)$, and $U_{i}^{\prime} s \quad$ new pseudonym from $C I D_{i}^{\text {new }}=B_{16} \oplus h\left(\left(A_{2} \oplus h\right.\right.$ $\left.\left.\left(I D_{i} \| H P W_{i}\right)\right) \| R_{s}\right)$. Then it computes $S K_{u}=h\left(R_{u} \oplus\right.$ $H P W_{i}\left\|R_{g}\right\| R_{s}$ ) to get the common session key.

After checking $B_{17}$, it stores $C I D_{i}^{\text {new }}$ and $A_{1}^{\text {new }}$ which are derived by calculating $A_{1}^{\text {new }}=B_{13} \oplus h\left(R_{u} \| H P W_{i}\right)$.

\section{Cryptanalysis of Fotouhi et al.'s Scheme}

This section shows that Fotouhi et al.'s scheme has some security drawbacks.

\subsection{Too Much Data Storage in Registration Phase}

In registration phase of Fotouhi et al.'s scheme, the gateway $G W_{j}$ must store data $\left\{S I D_{\mathrm{k}}, Q I D_{k}, N_{1}, R_{y}, h\left(R_{z}\right)\right\}$ for a sensor $S N_{k}$.

The gateway $G W_{j}$ also must store $\left\{C I D_{i}, I D_{i}, R_{x}, H P W_{i}\right\}$ for a sensor $U_{i}$

However, the scheme is oriented to multiple users and multiple servers, it will take up too much storage space for the gateway to store users and sensors registration data.

\subsection{Some Identities Data Missing in Submitted Messages in Authentication Phase}

This scheme cannot efficiently work because some identities data missing in submitted the messages. For example, at step 2 in authentication phase, the message:

$$
M_{2}=\left(Q I D_{k}, B_{5}, B_{6}, B_{7}, B_{8}, B_{9}\right)
$$

From the gateway $G W_{\mathrm{j}}$ to the sensor $S N_{K}$, due to the gateway identity $G I D_{\mathrm{j}}$ missing in $M_{2}$, the target sensor $S N_{K}$ does not know the message sent from $G I D_{\mathrm{j}}$. So, $S N_{K}$ cannot compute $s=h\left(S G_{K} \| G I D_{j}\right)$ without $G I D_{\mathrm{j}}$. Such, next operations cannot be executed.
Similarly, due to flaws in messages $M_{3}$ at step 3 in authentication phase:

$$
M_{3}=\left(B_{10}, B_{11}, B_{12}\right)
$$

From the sensor $S N_{K}$ to the gateway $G W_{j}$, due to the sensor temporary identity $Q I D_{k}$ and the user temporary identity $C I D_{i}$ missing in $M_{3}$. The gateway $G W_{j}$ does not know the message from target sensor $S N_{K}$, so the gateway $G W_{j}$ cannot compute $R_{y}^{\prime}=h\left(R_{y}\right)$ without $Q I D_{k}$. In addition, if $C I D_{i}$ is missing, the gateway $G W_{j}$ cannot update $C I D_{i}$ to $C I D_{i}^{\text {new }}$ by computing $\quad B_{13}=h\left(C I D_{i}^{\text {new }}\left\|h\left(R_{x}\right)\right\| G I D_{j}\right)$. Such, next operations cannot be executed.

Finally, due to flaws in messages $M_{4}$ at step 4 in authentication phase,

$$
M_{4}=\left(B_{13}, B_{14}, B_{15}, B_{16}, B_{17}\right)
$$

From the gateway $G W_{j}$ to the user $U_{i}$, the user $U_{i}$ cannot compute $C I D_{i}^{\text {new }}=B_{16} \oplus h\left(\left(A_{2} \oplus h\left(I D_{i} \| H P W_{i}\right)\right) \| R_{s}\right)$ without $A_{2}$. Due to the user $U_{i}$ store $A_{2}$ with the gateway identity $G I D_{\mathrm{j}}$, however the gateway identity $G I D_{\mathrm{j}}$ missing in $M_{4}$. So, the user $U_{i}$ cannot find $A_{2}$ by the gateway identity $G I D_{\mathrm{j}}$ to compute $C I D_{i}^{\text {new }}=B_{16} \oplus h\left(\left(A_{2} \oplus h\left(I D_{i} \| H P W_{i}\right)\right) \| R_{s}\right)$. Such, next operations cannot be executed.

\subsection{More Data are Needed Stored in Authentication Phase}

After the gateway $G W_{j}$ receives the response message $M_{3}$ sent by the target sensor $S N_{k}, G W_{j}$ starts to perform corresponding calculations:

$$
R_{Z}^{\prime}=R_{g} \oplus S \oplus B_{10}
$$

at step 4 .

But, it needs random number $R_{g}$ and $s$ previously generated by $G W_{j}$ at step 2. So, $G W_{j}$ must store $R_{g}$ and $Q I D_{k}$ at step 2. Unfortunately, $G W_{j}$ does not store $R_{g}$ with $Q I D_{k}$ at step 2.

Furthermore, at step 4 it needs random number $R_{u}$ and $H P W_{i}$ for the following computation

$$
S K_{g}=h\left(R_{u} \oplus H P W_{i}\left\|R_{g}\right\| R_{s}\right)
$$

But, at step $2 G W_{j}$ does not store $R_{u}$ and $H P W_{i}$.

To sum up, the authentication of Fotouhi et al.'s scheme cannot work without the storage of $R_{g}, Q I D_{k}, R_{u}$ and $H P W_{i}$ by $G W_{j}$ at step 2 .

\section{Improved Scheme}

In order to overcome the flaws of the Fotouhi et al.'s scheme, an improved scheme is proposed. Same as Fotouhi et al.'s scheme, the gateway $G W_{j}$, the sensor $S N_{k}$ and the user $U_{i}$ in the improved scheme want share a session key. But, the sensor $S N_{k}$ and the user $U_{i}$ cannot communicate directly. The proposed scheme contains the following three phases: 
Initialization phase, registration, phase and authentication phase.

\subsection{Initialization Phase}

This phase is as same as that of the Fotouhi et al.'s scheme. So, it is omitted.

\subsection{Registration of Sensor}

Each sensor $S N_{k}$ with its identity $S I D_{k}$ belongs to network identifier $N_{1}$. Also $S N_{k}$ and $G W_{\mathrm{j}}$ share a common secret key $S G_{k}=h\left(S I D_{k}\left\|G_{j}\right\| N_{1}\right), G W_{\mathrm{j}}$ selects a pseudo-identity $Q I D_{k}$ for $S N_{k}$ and injects $\left\{S I D_{k}, S G_{k}, G I D_{j}, Q I D_{k}\right\}$ into $S N_{k}$. $G W_{\mathrm{j}}$ also stores $\left\{S I D_{\mathrm{k}}, Q I D_{k}, N_{1}, G I D_{j}\right\}$ in its database. Then, the sensor will be installed in the intended place.

\subsection{Registration Phase for User}

A user, $U_{i}$ registers herself/himself to the gateway $G W_{\mathrm{j}}$ by the following operations:

Step 1. the $U_{i}$ selects an identity, $I D_{i}$ and the corresponding password, $P W_{i}$ and a secret random nonce $R_{0}$ to compute masked password:

$$
H P W_{\mathrm{i}}=h\left(P W_{\mathrm{i}} \| R_{0}\right)
$$

and sends $I D_{i}$ and $H P W_{i}$ to $G W_{\mathrm{j}}$ via a secure channel.

Step 2. If $I D_{\mathrm{i}}$ has already been unregistered, $G W_{\mathrm{j}}$ generates a pseudo-identity $C I D_{i}$ for $U_{i}$ and stores them with $I D_{i}$ and $H P W_{i}$ in its database. Then, it computes:

$$
A_{1}=h\left(C I D_{i}\left\|G I D_{\mathrm{j}}\right\| G_{\mathrm{j}}\right) \oplus H P W_{i}
$$

and sends $\left\{A_{1}, C I D_{i}, G I D_{j}\right\}$ to $U_{i}$ via a secure channel.

Step 3. $U_{i}$ calculates:

$$
A_{2}=h\left(I D_{i} \| P W_{i}\right) \oplus R_{0}
$$

and stores $\left\{A_{1}, A_{2}, C I D_{i}, G I D_{j}\right\}$ into his/her mobile device.

\subsection{Authentication Phase}

$U_{i}, G W_{\mathrm{j}}$, and $S N_{k}$ carry out mutual authentication to set up a session key. The detailed description of the authentication phase are as follows:

Step 1. the $U_{i}$ selects intended sensor $S N_{k}$ and insert $I D_{i}$ and $P W_{i}$ to the mobile device which calculates

$$
R_{0}=A_{2} \oplus h\left(I D_{i} \| P W_{i}\right), H P W_{i}=h\left(P W_{i} \| R_{0}\right) .
$$

Then, $U_{i}$ generates a random number $R_{u}$ and computes:

$$
\begin{gathered}
B_{1}=A_{1} \oplus H P W_{i} \oplus R_{u}, \\
B_{2}=S I D_{k} \oplus h\left(R_{u}\right), B_{3}=h\left(C I D_{i}\left\|G I D_{j}\right\| S I D_{k} \| R_{u}\right) .
\end{gathered}
$$

Then, $U_{i}$ sends the message $M_{1}=\left(C I D_{i}, G I D_{j}, B_{1}, B_{2}, B_{3}\right)$ to $G W_{\mathrm{j}}$.

Step 2. On receiving $G W_{\mathrm{j}}$ the message $M_{1}$ from $U_{i}, G W_{\mathrm{j}}$ computes:

$$
R_{u}=B_{1} \oplus h\left(C I D_{i}\left\|G I D_{j}\right\| G_{j}\right)
$$

and generates a random numbers $R_{g}$. Afterwards, $G W_{\mathrm{j}}$ calculates:

$$
S I D_{k}=B_{2} \oplus h\left(R_{u}\right), B^{*}=h\left(C I D_{i}\left\|G I D_{j}\right\| S I D_{k} \| R_{u}\right)
$$

check $B_{3}^{*} \stackrel{?}{=} B_{3}$, if it holds, continue the operation, otherwise terminate the session. Then, $G W_{\mathrm{j}}$ generates a new pseudonym $Q I D_{k}^{\text {new }}$ for the sensor $S N_{k}$ and a new pseudonym $C I D^{\text {new }}{ }_{i}$ for the user $U_{i}$. Then, $G W_{\mathrm{j}}$ computes

$$
\begin{gathered}
S G_{k}=h\left(S_{k}\left\|D_{j}\right\| N_{1}\right), \\
B_{4}=\left(h\left(C I D_{i}^{\text {new }}\left\|G I D_{j}\right\| G_{j}\right) \| C I D_{i}^{\text {new }}\right) \oplus R_{u}, \\
B_{5}=\left(R_{u} \| R_{g}\right) \oplus h\left(S G_{k} \| C I D_{i}\right), \\
B_{6}=h\left(B_{4}\left\|B_{5}\right\| C I D_{i}^{\text {new }}\right), \\
B_{7}=Q I D^{\text {new }} \oplus h\left(R_{u} \| R_{g}\right), \\
B_{8}=h\left(Q I D_{k}\left\|C I D_{i}\right\|\left(R_{u} \| R_{g}\right)\left\|B_{7}\right\| B_{4}\right)
\end{gathered}
$$

and $G W_{\mathrm{j}}$ stores $C I D_{i}^{\text {new }}$ with $C I D_{i}$, stores $Q I D_{k}^{\text {new }}$ with $\left\{S I D_{k}, Q I D_{k}, S G_{k}, N_{1}\right\}$. Then, $G W_{\mathrm{j}}$ sends the message $M_{2}=\left(G I D_{j}, Q I D_{k}, C I D_{i}, B_{4}, B_{5}, B_{6}, B_{7}, B_{8}\right)$ to the sensor $S N_{k}$.

Step 3. After receiving the message $M_{2}$, the sensor $S N_{k}$ calculates:

$$
\begin{gathered}
R_{u} \| R_{g}=B_{5} \oplus h\left(S G_{k} \| C I D_{i}\right), \\
Q I D_{k}^{\text {new }}=B_{7} \oplus h\left(R_{u} \| R_{g}\right) \\
B_{8}^{*}=h\left(Q I D_{k}\left\|C I D_{i}\right\|\left(R_{u} \| R_{g}\right)\left\|B_{7}\right\| B_{4}\right)
\end{gathered}
$$

Then check $B_{8}^{*} \stackrel{?}{=} B_{8}$, if it holds, $S N_{k}$ generates a random number $R_{S}$ and computes the common session key:

$$
S K_{s}=h\left(R_{u}\left\|R_{g}\right\| R_{s}\right)
$$

Then, $S N_{k}$ generates:

$$
B_{9}=R_{s} \oplus Q I D_{k}^{n e w}, B_{10}=h\left(Q I D_{k}^{\text {new }} \| s k_{s}\right)
$$

and sends $M_{3}=\left(Q I D_{k}, C I D_{i}, B_{4}, B_{5}, B_{6}, B_{9}, B_{10}\right)$ to the $G W_{\mathrm{j}}$, then $\quad S N_{k} \quad$ updates $\left\{S I D_{k}, Q I D_{k}, S G_{k}, G I D_{j}\right\} \quad$ to $\left\{S I D_{k}, Q I D_{\mathrm{k}}^{\text {new }}, S G_{k}, G I D_{j}\right\}$.

Step 4. On receiving the response message $M_{3}$ from sensor $S N_{k}$, the gateway $G W_{\mathrm{j}}$ first find $Q I D_{k}^{\text {new }}$ and $C I D^{\text {new }}$ by $Q I D_{k}$ and $C I D_{i}$ in $M_{3}$. Then, $G W_{\mathrm{j}}$ computes:

$$
R_{s}=B_{9} \oplus Q I D_{k}^{\text {new }}, B_{6}^{*}=h\left(B_{4}\left\|B_{5}\right\| C I D_{i}^{\text {new }}\right) \text {. }
$$


and checks $B_{6}^{*} \stackrel{?}{=} B_{6}$ if it holds, $G W_{\mathrm{j}}$ computes:

$$
R_{u} \| R_{g}=B_{5} \oplus h\left(S G_{k} \| C I D_{i}\right)
$$

and the common session key $s k_{\mathrm{g}}$ and $B_{10}^{*}$ :

$$
s k_{\mathrm{g}}=h\left(R_{u}\left\|R_{g}\right\| R_{s}\right), B_{10}^{*}=h\left(Q I D_{k}^{\text {new }} \| s k_{g}\right)
$$

and checks $B_{10}^{*} \stackrel{?}{=} B_{10}$ if it holds, then, $G W_{\mathrm{j}}$ computes:

$$
B_{11}=\left(R_{g} \| R_{s}\right) \oplus R_{u}, B_{12}=h\left(G I D_{j}\left\|B_{4}\right\| B_{5} \| s k_{g}\right) .
$$

and $G W_{\mathrm{j}}$ sends the message $M_{4}=\left(C I D_{i}, G I D_{j}, B_{4}, B_{5}, B_{11}, B_{12}\right)$ to the user $U_{i}$. Then $G W_{\mathrm{j}}$ updates $\left\{S I D_{k}, Q I D_{k}, S G_{k}, N_{1}\right\}$ to $\left\{S I D_{k}, Q I D_{\mathrm{k}}^{\text {new }}, S G_{k}, N_{1}\right\}$ and updates $\left\{C I D_{i}, I D_{i}, H P W_{i}\right\}$ to $\left\{C I D_{i}^{\text {new }}, I D_{i}, H P W_{i}\right\}$.

Step 5. On receiving the response message $M_{4}$ from the gateway $G W_{\mathrm{j}}, U_{i}$ 's mobile device calculates:

$$
R_{g} \| R_{s}=B_{11} \oplus R_{u}, s k_{u}=h\left(R_{u}\left\|R_{g}\right\| R_{s}\right)
$$

to get the common session key. Then, $U_{i}$ 's mobile device computes:

$$
B_{12}^{*}=h\left(G I D_{j}\left\|B_{4}\right\| B_{5} \| s k_{u}\right)
$$

and check $B_{12}^{*} \stackrel{?}{=} B_{12}$, if it holds, $U_{i}$ 's mobile device calculates

$$
\begin{gathered}
h\left(C I D_{i}^{\text {new }}\left\|G I D_{j}\right\| G_{j}\right) \| C I D_{i}^{\text {new }}=B_{4} \oplus R_{u}, \\
A_{1}^{\text {new }}=h\left(C I D_{i}^{\text {new }}\left\|G I D_{j}\right\| G_{j}\right) \oplus H P W_{i} .
\end{gathered}
$$

After that, $U_{i}$ 's mobile device updates $\left\{A_{1}, A_{2}, C I D_{i}, G I D_{j}\right\}$ to $\left\{A_{1}^{\text {new }}, A_{2}, C I D_{i}^{\text {new }}, G I D_{j}\right\}$.

\section{Security Analysis}

\subsection{Informal Security Analysis}

This section shows that the improved scheme is well protected against relevant security threats. Firstly, like Fotouhi et al.'s scheme [15], Firstly, the improved scheme has absorbed the advantages of Fotouhi et al.'s scheme that can well resist the opponent's replay attacks and tracking attacks by updating the temporary identities of users and sensors, and also can avoid the verification of timestamps by the gateway stores the temporary identities which updated by users and sensors. next we will discuss separately that the improved protocol effectively solves the flaws about too much data storage in registration phase, some identities data missing in submitted messages in authentication phase, and more data are needed stored in authentication phase.

Firstly, in registration phase of the improved scheme, the sensor $S N_{k}$, the gateway $G W_{\mathrm{j}}$ and the user $U_{i}$ just need to store:

$$
\begin{gathered}
\left\{S I D_{k}, S G_{k}, G I D_{j}, Q I D_{k}\right\} \\
\left\{S I D_{\mathrm{k}}, Q I D_{k}, N_{1}, G I D_{j}\right\},\left\{C I D_{i}, I D_{i}, H P W_{i}\right\}
\end{gathered}
$$

$$
\left\{A_{1}, A_{2}, C I D_{i}, G I D_{j}\right\}
$$

separately in their database. However in registration phase of the Fotouhi et al.'s scheme, the sensor $S N_{k}$, the gateway $G W_{\mathrm{j}}$ and the user $U_{i}$ needs to store:

$$
\begin{gathered}
\left\{S I D_{k}, S G_{k}, G I D_{j}, R_{y}, R_{z}, Q I D_{k}\right\} \\
\left\{S I D_{\mathrm{k}}, Q I D_{k}, N_{1}, R_{y}, h\left(R_{z}\right)\right\},\left\{C I D_{i}, R_{x}, I D_{i}, H P W_{i}\right\} \\
\left\{A_{1}, A_{2}, A_{3}, C I D_{i}, G I D_{j}\right\}
\end{gathered}
$$

in their database. Obviously, we can find that the amount of data stored in the sensor $S N_{k}$, the gateway $G W_{\mathrm{j}}$ and the user $U_{i}$ in Fotouhi et al.'s scheme in the registration phase will be much larger than the amount of data stored in the improved scheme.

Secondly, in authentication phase of Fotouhi et al.'s scheme, some identities data missing in submitted messages between the sensor $S N_{k}$, the gateway $G W_{\mathrm{j}}$ and the user $U_{i}$, which will make Fotouhi et al.'s scheme unable to be effectively implemented. So, we need to fill in the missing data in submitted messages between the sensor $S N_{k}$, the gateway $G W_{\mathrm{j}}$ and the user $U_{i}$ to ensure that Fotouhi et al.'s scheme can be executed normally.

Finally, there is more data need to be stored in authentication phase of Fotouhi et al.'s scheme. As analyzed by 3.3 in Cryptanalysis of Fotouhi et al.'s scheme above that the gateway $G W_{j}$ need to store $R_{g}, Q I D_{k}, R_{u}$ and $H P W_{i}$ at step 2. in authentication phase of the improved scheme. Due to Fotouhi et al.'s scheme is oriented to multiple users and multiple servers, it means that a large amount of intermediate data needs to be stored when the gateway assists multiple users and multiple servers to communicate. However, in the authentication phase of our improved scheme, we can reduce the amount of data stored by the gateway $G W_{\mathrm{j}}$ by adding $B_{4}$, $B_{5}$, and $B_{6}$.

$$
\begin{gathered}
B_{4}=\left(h\left(C I D_{i}^{\text {new }}\left\|G I D_{j}\right\| G_{j}\right) \| C I D_{i}^{n e w}\right) \oplus R_{u}, \\
B_{5}=\left(R_{u} \| R_{g}\right) \oplus h\left(S G_{k} \| C I D_{i}\right), B_{6}=h\left(B_{4}\left\|B_{5}\right\| C I D^{\text {new }}{ }_{i}\right),
\end{gathered}
$$

to the messages $M_{2}$ and $M_{3}$. The gateway $G W_{\mathrm{j}}$ sends a message $M_{2}$ to the sensor $S N_{k}$ in step 2 of the verification phase. After receiving the message $M_{2}$, the $S N_{k}$ will return $B_{4}$, $B_{5}$, and $B_{6}$ to the $G W_{\mathrm{j}}$ at step 3 in the authentication phase. In these two steps, $B_{4}$ and $B_{5}$ are used to transmit the protected information, while $B_{6}$ can be used at step 4 in the authentication phase. when the information $B_{4}$ and $B_{5}$ send back by the sensor $S N_{k}$ to the gateway $G W_{\mathrm{j}}, B_{6}$ can be used to verify $B_{4}$ and $B_{5}$ to prevent the message $M_{3}$ from being changed during transmission. This method not only enables the efficient execution of the program, but also saves the space for storing some intermediate data by the gateway $G W_{\mathbf{j}}$.

In summary, the improved scheme completely overcomes the shortcomings of Fotouhi et al.'s scheme and retains the advantages of Fotouhi et al.'s scheme. In the improved scheme, neither the sensor $S N_{k}$ nor the gateway $G W_{\mathrm{j}}$ nor the user $U_{i}$ need to store large amounts of data in the registration phase. Meanwhile, less intermediate data needs to be stored 
by the gateway $G W_{\mathrm{j}}$ in the authentication phase. Therefore, more storage space can be saved in our improved scheme.

\subsection{Formal Security Analysis}

5.2.1 Simulation for security verification with the AVISPA tool

We performed a formal security verification of the proposed scheme utilizing AVISPA simulation tool $[16,17]$ to evaluate the safety of the authentication protocol against MITM and replay attacks, which is widely accepted for formal security analysis [18-20]. To perform AVISPA simulation tool, the environment and the session of security protocol must be implemented using the High Level Protocols Specification Language (HLPSL).

\subsubsection{HLPSL specifications}

We considered three basic roles: user $U_{i}$, gateway $G W_{\mathrm{j}}$, and sensor $S N_{k}$. Then, we present session and environment utilizing HLPSL in Figure 1, which contains the security goals. The role specifications of $U_{i}, G W_{\mathrm{j}}$, and $S N_{k}$ are as shown in Figures 2-4.

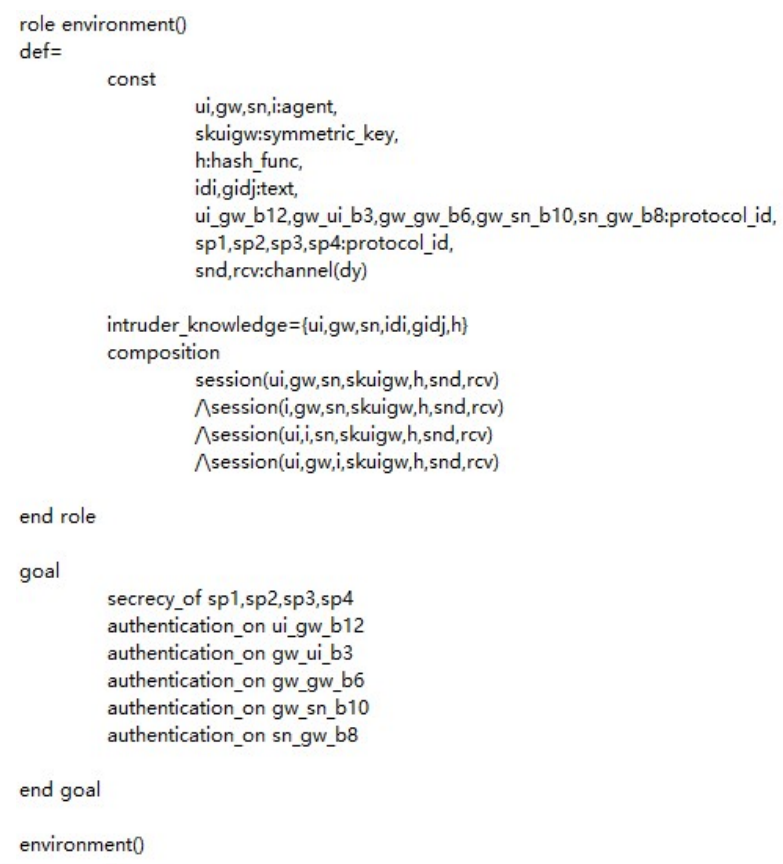

Figure 1: Role for environment and session in HLPSL.

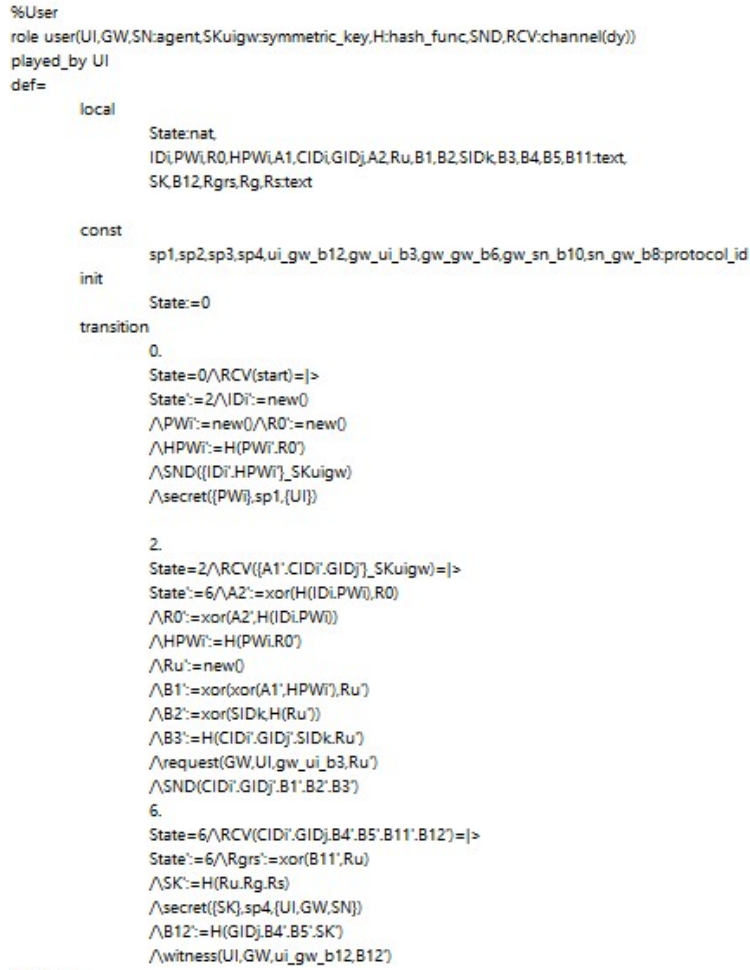

Figure 2: Role specification for user

\&GateWa

role

gateway (UI, GW, SN: agent, SKuigw: symmetric_key, H: hash

func, SND, RCV: channel (dy))

played_by GW

$$
\text { local }
$$

State: nat,

IDi, HPWi , CIDi, GIDj , A1 , Gj , B1 , B2 , B3 , Ru , Rg , SIDk, SGk, N1 B4, B5, B6, B7, B8 : text

PWi, QIDk, B9 , B10 , Rs , Rurg, SK, B11, B12, CIDinew, QIDknew: text

const

sp1,sp2,sp3,sp4, ui_gw_b12,gw_ui_b3, gw_gw_b6,gw_sn_b 10, sn_gw_b8:protocol_i

State $:=1$

transition

State $=1 / \operatorname{RCV}\left(\left\{\mathrm{IDi}^{\prime} \cdot\right.\right.$ HPWi' $\}$ SKuigw $)=1>$

State' $:=3$ TCIDi $^{\prime}:=$ new $(1)$

八GIDj' : =new()

$\widehat{\wedge A 1}:=\operatorname{xor}\left(\mathrm{H}\left(\mathrm{CIDi}^{\prime}\right.\right.$.GIDj'.Gj), HPWi')

secret $\{$ Gj\}, sp2, $\{\mathrm{GW}\})$

$\bigwedge \operatorname{SND}\left(\left\{A 1^{\prime}\right.\right.$. CIDi'.GIDj'\}_SKuigw)

State=3/RCV $\left(\operatorname{CIDi}{ }^{\prime} \cdot\right.$ GIDj' $^{\prime} \cdot B 1^{\prime} \cdot B 2^{\prime} \cdot$ B3 $\left.^{\prime}\right)=\mid>$

State $\left.{ }^{\prime}:=5 / \mathrm{Ru}^{\prime}:=\operatorname{xor}\left(B 1^{\prime}, \mathrm{H}(\mathrm{CIDi})^{\prime} . \mathrm{GIDj}{ }^{\prime} . \mathrm{Gj}\right)\right)$

$八 \mathrm{Rg} \mathbf{l}^{\prime}=$ new ( )

$\bigwedge \mathrm{SIDk}^{\prime}:=\operatorname{xor}\left(\mathrm{B}^{\prime}{ }^{\prime}, \mathrm{H}\left(\mathrm{Ru}^{\prime}\right)\right)$

八B3 ${ }^{\prime}:=$ H $^{\prime}$ (CIDi'.GIDj'.SIDk'.Ru')

八witness(GW,UI,gW_ui_b3,Ru')

八SGk': $=\mathrm{H}(\mathrm{SIDk} \cdot \mathrm{Gj} \cdot \overline{\mathrm{N}} 1)$

八CIDinew': : =new(

八QIDknew': =new( )

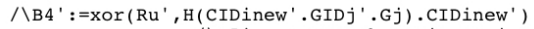

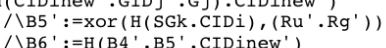

八request (GW, GW, gw gw b6, B6 ')

$\wedge \mathrm{B}^{\prime}:=\operatorname{xor}\left(\mathrm{QIDknew}^{\prime}, \mathrm{H}^{\mathrm{H}}\left(\mathrm{Ru}^{\prime} \cdot \mathrm{Rg}^{\prime}\right)\right)$

$\left.\left(\mathrm{Ru}^{\prime} \cdot \mathrm{Rg}^{\prime}\right) . \mathrm{B}^{\prime} \cdot \mathrm{B}^{\prime}{ }^{\prime}\right)$ $\backslash \mathrm{B}^{\prime}:=\mathrm{H}$ (OIDk.CIDi'

八request ( SN, GW, sn_gw_b8, B8' )

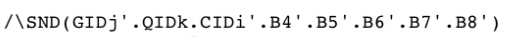

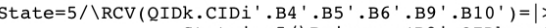
State $^{\prime}:=5 / \mathrm{Rs}^{\prime}:=\operatorname{xor}\left(\mathrm{Bg}^{\prime}{ }^{\prime}\right.$, OIDknew $)$ 八Rurg': $:=$ xor (B5 $\left.{ }^{\prime}, \mathrm{H}^{\prime}\left(\mathrm{SGk} . \mathrm{CIDi}^{\prime}\right)\right)$ $八 \mathrm{~B}^{\prime}:=\mathrm{H}(\mathrm{B} 4 . \mathrm{B} 5$. CIDinew) 八witness (GW, GW, gw_gw_b6, B6') 八SK':=H(Rurg'.Rs') 八B10 $:=$ :H( QIDknew.SK) 八witness (GW, SN, gw_sn_b10,B10 八B11': $:=\operatorname{xor}\left(\mathrm{Ru},\left(\mathrm{Rg}^{\prime} \mathrm{Rs}^{\prime}\right)\right)$ ASND(CIDi'.GIDj.B4'.B5'.B11'.B12')

end role

Figure 3: Role specification for gateway 


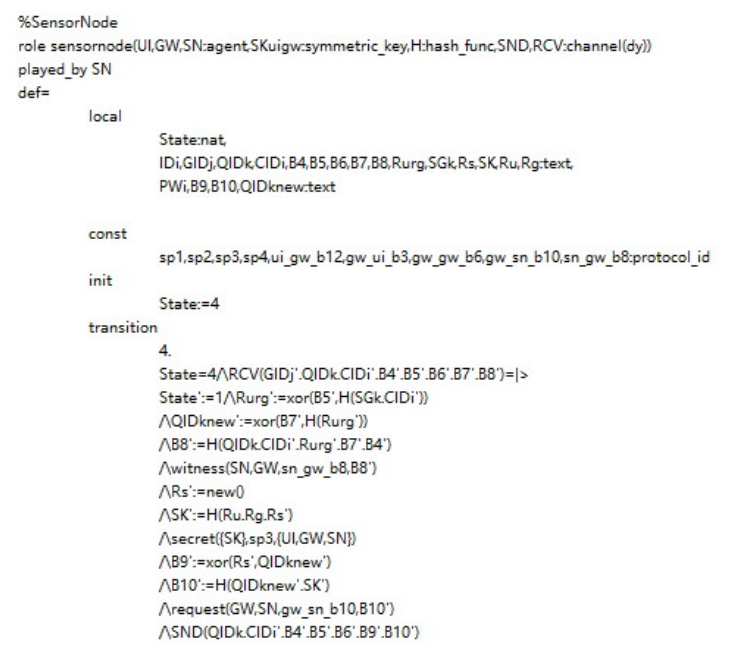

Figure 4: Role specification for sensor

\subsubsection{AVISPA Simulation Result}

We show the AVISPA results to verify the safety of the proposed scheme using OFMC and CL-AtSe. The OFMC and the CL-AtSe checks whether the proposed scheme is safe. Consequently, Figure 5 shows that the proposed scheme is secure though AVISPA simulation.

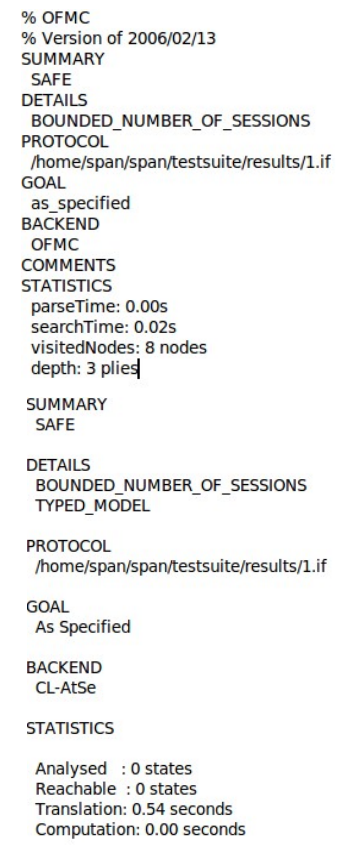

Figure 5: Analysis of AVISPA simulation using OFMC and CL-AtSe

\section{Conclusion}

In this paper, we find that Fotouhi et al.'s scheme is vulnerable to several weaknesses in the phase of registration and authentication. To overcome the shortcomings of Fotouhi et al.'s scheme, we propose an improved scheme. The improved scheme not only completely overcomes the shortcomings of Fotouhi et al.'s scheme but also retains the advantages of Fotouhi et al.'s scheme. Otherwise, the improved scheme has less computation cost.

\section{Acknowledgements}

This work is supported by the Applied Basic and Advanced Technology Research Programs of Tianjin (No. 15JCYBJC15900).

\section{References}

[1] Z.-Y. Wu, Y.-C. Lee, F. Lai, H.-C. Lee, Y. Chung, A secure authentication scheme for telecare medicine information systems $[\mathrm{J}]$. Journal of Medical Systems, 2012, 36(3): 1529-1535.

[2] D. He, N. Kumar, J. Chen, C.C. Lee, N. Chilamkurti, S.S. Yeo, Robust anonymous authentication protocol for health-care applications using wireless medical sensor networks[J]. Multimedia Systems, 2015, 21(1): 49-60.

[3] D. He, S. Zeadally, L. Wu, Certificateless public auditing scheme for cloud-assisted wireless body area networks, IEEE Systems Journal 12 (1) (2015) 64-73.

[4] S. Kumari, X. Li, F. Wu, A.K. Das, H. Arshad, M.K. Khan, A user friendly mutual authentication and key agreement scheme for wireless sensor networks using chaotic maps[J]. Future Generation Computer Systems 2016, 63: 56-75.

[5] D. He, S. Zeadally, N. Kumar, J.H. Lee, Anonymous authentication for wireless body area networks with provable security[J]. IEEE Systems Journal, 2016, 11(4): 2590-2601.

[6] P. Gope, T. Hwang, A realistic lightweight anonymous authentication protocol for securing real-time application data access in wireless sensor networks, IEEE Trans[J]. Industrial Electronics, 2016, 63(11): 7124-7132.

[7] Y. Lu, L. Li, H. Peng, Y. Yang, An energy efficient mutual authentication and key agreement scheme preserving anonymity for wireless sensor networks[J]. Sensors, 2016, 16(6): 837.

[8] F. Wu, L. Xu, S. Kumari, X. Li, An improved and anonymous two-factor authentication protocol for health-care applications with wireless medical sensor networks[J]. Multimedia Systems, 2017, 23(2): 195-205.

[9] R. Amin, S.H. Islam, G. Biswas, M.K. Khan, N. Kumar, A robust and anonymous patient monitoring system using wireless medical sensor networks[J]. Future Generation Computer Systems, 2018, 80: 483-495.

[10] F. Wei, P. Vijayakumar, J. Shen, R. Zhang, L. Li, A provably secure password-based anonymous authentication scheme for wireless body area networks[J]. Computers \& Electrical Engineering, 2018, 65: 322-331.

[11] M. Wazid, A.K. Das, A.V. Vasilakos, Authenticated key management protocol for cloud-assisted body area sensor networks $[\mathrm{J}]$. Journal of Network and Computer Applications, 2018, 123: 112-126.

[12] M. Shuai, B. Liu, N. Yu, L. Xiong, C. Wang, Efficient and privacy-preserving authentication scheme for wireless body area networks[J]. Journal of Information Security and Applications, 2020, 52: 102499.

[13] A. Gupta, M. Tripathi, T.J. Shaikh, A. Sharma, A Lightweight Anonymous User Authentication and Key 
Establishment Scheme for Wearable Devices[J]. Computer Networks, 2018, 149: 29-42.

[14] M. Wazid, A.K. Das, A.V. Vasilakos, Authenticated key management protocol for cloud-assisted body area sensor networks[J]. Journal of Network and Computer Applications, 2018, 123: 112-126.

[15] M. Fotouhi, M. Bayat, A.K. Das, H.A. Far, S.M. Pournaghi, M.A. Doostari, A lightweight and secure two-factor authentication scheme for wireless body area networks in health-care IoT $[\mathrm{J}]$. Computer Networks, 2020, 177(177): 1073333.

[16] AVISPA. Automated Validation of Internet Security Protocols and Applications. Available online: http://www.avispa-project.org/(accessed on 6 May 2019).

[17] SPAN: A Security Protocol Animator for AVISPA. Available online: http://www.avispa-project.org/(accessed on 6 May 2019).

[18] K. Park, Y. Park, A.G. Reddy, A.K. Das. Provably secure and efficient authentication protocol for roaming service in global mobility networks[J]. IEEE Access, 2017, 5: 25110-25125.

[19] K. Park, Y. Park, A.G. Reddy, A.K. Das 2PAKEP: Provably secure and efficient two-party authenticated key exchange protocol for mobile environment[J]. IEEE Access, 2018, 6: 30225-30241.

[20] S. Yu, J. Lee, K. Lee, K. Park, Y. Park. Secure authentication protocol for wireless sensor networks in vehicular communications[J]. Sensors, 2018, 18: 3191. 